\title{
EVALUATION OF THE CANADIAN CT HEAD RULE FOR ANALYZING DIAGNOSTIC ACCURACY IN PATIENTS WITH MINOR HEAD TRAUMA
}

\author{
Volkan Kızılgöz ${ }^{1}$, Sema Avcı ${ }^{2}$, Ali Kemal Sivrioğlu ${ }^{3}$, Hasan Aydın ${ }^{4}$, Türkhun Çetin ${ }^{1}$, Berihat Kızılgöz ${ }^{2}$ \\ Correspondence: volkankizilgoz@gmail.com \\ ${ }^{I}$ Department of Radiology Faculty of Medicine Kafkas University, Kars, Turkey. \\ ${ }^{2}$ Department of Emergency Medicine Harakani State Hospital, Kars, Turkey. \\ ${ }^{3}$ Department of Radiology Faculty of Medicine Kocaeli University, Kocaeli, Turkey. \\ ${ }^{4}$ Department of Radiology Abdurrahman Yurtaslan Ankara Oncology Research and Training Hospital, Ankara, Turkey.
}

\section{Article History:}

Received: December 10, 2018

Accepted: February 21, 2019

Published: July 1, 2019

Cite this as:

Kızllgöz V, Avcı S, Sivrioğlu AK,

Aydin H, Çetin T, Kızllgöz B.

Evaluation of the canadian $c t$

head rule for analyzing

diagnostic accuracy in patients

with minor head trauma. Malang

Neurology Journal; 2019.5:68-

75. DOI:

http://dx.doi.org/10.21776/ub.mnj

.2019 .005 .02 .4

\section{ABSTRACT}

Background: Head computed tomography (CT) scans, which provide reliable information to assessor rule out neurological abnormalities and injuries, should be used judiciously to minimize radiation exposure and cost.

Objective: The aim of this study was to evaluate the widely used Canadian CT Head Rule (CCHR) to determine the necessity of head CT scans in patients with minor head trauma.

Methods: This retrospective study included 913 patients with head trauma who were admitted to Harakani State Hospital between June 2014 and December 2017. The patient data were reevaluated according to CCHR and compared with the radiological findings.

Results: There were a total of 556 patients fulfilling the inclusion criteria with a male/female ratio of 2.95 and a mean age of $37 \pm 4.24$ years. The most common injury mechanism of head trauma was motor vehicle accidents. Chi square tests were applied for statistical calculations. The sensitivity, specificity, positive predictive value (PPV), negative predictive value (NPV), and accuracy of the CCHR were $93 \%, 88 \%, 88 \%, 94 \%$, and $90 \%$, respectively.

Conclusion: CCHR was highly useful for patients with minor head trauma who could benefit from head CT evaluation with high sensitivity and specificity.

Keywords: Canadian computed tomography head rule, Mild traumatic brain injury, Traumatic brain injury, Brain injuries, Emergency service

\section{Introduction}

Traumatic brain injury is one of the most common causes of emergency department visits. ${ }^{1}$ Glasgow Coma Scale (GCS) is a widely used method for the classification of the neurological status of patients with trauma, majority of whom suffer from minor head injuries. ${ }^{2}$ A patient with amnesia, disorientation, a history of loss of consciousness, and a GCS score of 13 to 15 is diagnosed as having "minor head injury"., ${ }^{3,4}$ The high incidence rates of minor head injuries are significant not only due to their long-term neurological impact on patients but also because of the substantial economic burden they place on healthcare services and the society. ${ }^{5,6}$ Non-contrast head computed tomography (CT) has become the standard imaging method to detect brain injuries in patients with head traumas. ${ }^{7} \mathrm{CT}$ is a fast and highly sensitive technique for the detection of acute brain hemorrhages and skull fractures ${ }^{1,8}$ and costs less than magnetic resonance imaging. ${ }^{1}$ Conversely, the cost and ionizing radiation exposure associated with head CT are the two most important disadvantages. ${ }^{1,2,45}$ The Canadian CT Head Rule (CCHR) is one of the most important criteria to determine the necessity of head CT in patients with minor head traumas. Clinicians in emergency departments need reliable criteria to avoid malpractice, minimize patient exposure to radiation, and avoid unnecessary healthcare expenses. Smits et al. found that the application of CCHR led to approximately $\$ 120$ million in cost savings, a substantial amount, in the United States. ${ }^{5}$

The objective of this study was to analyze CCHR to identify patients who could benefit the most from CT from those with minor head traumas. Additionally, we examined the relationship between radiological findings and GCS scores to elucidate the mechanisms of minor head traumas.

\section{Methods}

\section{CCHR}

In this study, we aimed to investigate the utility of CCHR developed by Stiell et al. in determining the necessity for head CT scans for minor head traumas. CCHR defines patients with minor head injuries as those with a history of amnesia, loss of consciousness or disorientation, and a GCS 
score between 13 and 15. CCHR suggests head CT in patients with minor head traumas who fulfill one of the following criteria: (1) patients with a GCS score of $<15$ at two h after injury, (2) patients with a suspected open or

depressed skull fracture, (3) patients with any sign of a basal skull fracture (hemotympanum, raccoon eyes, otorrhea or rhinorrhea, and Battle's sign), (4) patients with two or more episodes of vomiting, (5) patients aged more than 65 years, (6) persistent retrograde amnesia of greater than $30 \mathrm{~min}$, or (7) patients with a dangerous injury mechanism. Patients with a dangerous mechanism included those struck by a vehicle, occupants ejected from a motor vehicle, and those who fell from an elevation of more than three feet or five steps. Patients fulfilling the first five parameters are grouped as high risk and those fulfilling the last two parameters are classified as medium risk for minor head trauma. In addition, this rule is not applicable if the patient is not a trauma case, GCS score is lower than 13, patient is younger than 16 years of age, the patient is using coumadin or has a bleeding disorder, or the patient has an open skull fracture. ${ }^{4}$

\section{Patients}

The medical records of 913 patients with head traumas, who were admitted to Harakani State Hospital from June 2014 to December 2017, were assessed according to CCHR. Of these, 98 patients with major head traumas, 185 patients who were 16 years of age or younger, 45 patients fulfilling the exclusion criteria for CCHR, and 29 patients with missing data were excluded from the study; consequently, 556 patients (age, $37 \pm 4.24$ years) with sufficient data for evaluation according to CCHR were included into the study. This study was approved by the institutional ethics committee, which waived informed consent owing to the retrospective study design.

\section{Interpretation of patient data}

All head CT scans were performed based on the combined decision reached by at least one general practitioner and one emergency specialist in the emergency department. To elucidate the approach of each clinician and their tendency to use CCHR, the patients were grouped into those who did and did not undergo head CT scans. The patients were also grouped into those who needed and did not need a head CT according to CCHR (Table 1) and according to the risk level by CCHR (high risk, Table 2; medium risk, Table 3). The study parameters between the two classifications were compared. The patient distribution based on CCHR is presented in Figure 1.

The diagnostic power of CCHR was investigated by comparing the CCHR criteria with positive findings obtained by the head CT scans. Radiology reports of CT scans of the patients were used as the gold standard to determine whether CCHR was successful in predicting positive CT findings.

The diagnostic power of CCHR was assessed using all the positive criteria of the CCHR and specific positive CT findings. Stiell et al. included calvarial fractures as a bone fracture finding of CCHR. The first approach included assessing facial bone and calvarial bone fractures and brain parenchymal and extraparenchymal findings, whereas the second approach was identical to that used by Stiell et al. and excluded facial bone fractures. The third approach included only the parenchymal and extraparenchymal findings. The diagnostic utility of these subclassifications were analyzed according to sex and specific GCS scores. The distribution of patients according to specific GCS scores is presented in Table 4.

Positive CT findings included subdural hematoma, epidural hematoma, parenchymal contusion of the brain, calvarial fracture, (5) intraventricular hemorrhage, subarachnoid hemorrhage, cerebral edema, pneumocephaly, facial fracture, hemorrhage in paranasal sinuses, and axonal injury (Figure 2).

The patients were also grouped according to the injury mechanism as follows: (1) driver (ejected from the vehicle or head crushed) in a 4-wheel motor vehicle, (2) occupant (ejected from the vehicle or head-crushed) in a 4-wheel vehicle, (3) pedestrian stuck by a 4-wheel motor vehicle, (4) driver falling or ejected from a 2- or 3-wheel motor vehicle, (5) occupant falling or ejected from a 2- or 3-wheel vehicle, (6) pedestrian stuck by a 2- or 3-wheel motor vehicle, (7) fall accident, (8) assault by fist, (9) assault by a hard object, (10) hard object fallen onto head, (11) head crashed onto a hard object, (12) animal attack onto head, (13) gunshot (which does not directly penetrate the brain parenchyma), and (14) assault by sudden push or shake. All of the patients who were pedestrians struck by a 2- or 3wheel motor vehicle were determined to have major head traumas, therefore there was no patient minor head trauma to be shown in the graphics and tables related with this injury mechanism (Table 5).

The distribution of the injury mechanisms according to the need for head CT was determined based on CCHR (Figure 3 ), the high-risk and medium-risk criteria of CCHR (Figure 4), and GCS scores (Figure 5).

\section{Statistical analysis}

All statistical analyses were performed using the MedCalc software (Acacialaan, Ostend, Belgium). The diagnostic power of CCHR was calculated by the chi-square test. The sensitivity, specificity, positive predictive value (PPV), negative predictive value (NPV), and diagnostic accuracy of CCHR were calculated using the radiology reports of CTs as the gold standard. Data distribution of the patients according to specific CT findings and injury mechanisms were presented as bar graphs, and differences among the injury mechanisms were examined using the chi-square test. A $p$ value of $<0.05$ was considered statistically significant for all analyses.

\section{Results}

In total, 556 patients who fulfilled the eligibility criteria were included in the study. Among these, head CT scans were obtained in 312 patients. According to CCHR, 374 of the study patients did not require head CT scans, and 160 of these patients underwent unnecessary CT scans (Table 1). Figure 1 shows the distribution of patients according to the specific CCHR criteria. When solely the high-risk and medium-risk criteria were accepted in CCHR, 111 and 71 patients required head CT scans, respectively (Tables 2 and 3 , respectively). 
Table 1. Distribution of head CTs according to the CCHR.

\begin{tabular}{cccc}
\hline & Head CT performed (n) & Head CT not performed (n) & Total (n) \\
\hline $\begin{array}{c}\text { CT necessary according to the } \\
\text { CCHR (n) }\end{array}$ & 152 & 30 & 182 \\
$\begin{array}{c}\text { CT unnecessary according to } \\
\text { the CCHR (n) }\end{array}$ & 160 & 214 & 374 \\
\hline Total (n) & 312 & 244 & 556 \\
\hline
\end{tabular}

CCHR, Canadian CT Head Rule; CT, computed tomography

\section{The distribution of CCHR criteria}

Patients with dangerous mechanism

Persistent retrograde amnesia of greater than 30 minutes

Patients older than 65 years

Patients with $\geq 2$ episodes of vomiting

Patients with any sign of basal skull fracture

Patients with suspected open or depressed skull fracture

Patients with GCS score $<15$ at 2 hours after injury

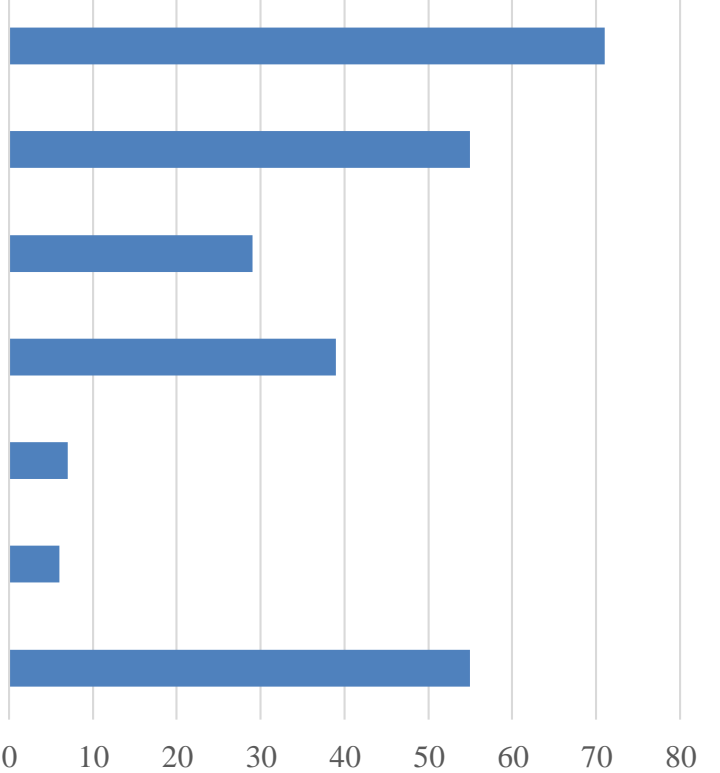

Figure 1. The frequencies of specific CCHR criteria within the study population. Some patients fulfilled more than one CCHR criterion.

Table 2. Distribution of head CTs according to the high-risk CCHR criteria.

\begin{tabular}{cccc}
\hline & Head CT performed (n) & $\begin{array}{c}\text { Head CT not performed } \\
(\mathbf{n})\end{array}$ & Total (n) \\
\hline $\begin{array}{c}\text { CT necessary according to } \\
\text { the high-risk CCHR } \\
\text { criteria (n) }\end{array}$ & 100 & 11 & 111 \\
$\begin{array}{c}\text { CT unnecessary according } \\
\text { to the high-risk CCHR } \\
\text { criteria (n) }\end{array}$ & 212 & 233 & 445 \\
\hline Total (n) & 312 & 244 & 556 \\
\hline
\end{tabular}

CCHR, Canadian CT Head Rule; CT, computed tomography

Table 3. Decision of head CT of physicians in emergency department according to medium-risk CCHR criteria.

\begin{tabular}{cccc}
\hline & Head CT performed (n) & $\begin{array}{c}\text { Head CT not performed } \\
(\mathbf{n})\end{array}$ & Total (n) \\
\hline $\begin{array}{c}\text { CT necessary according to } \\
\text { the medium-risk CCHR } \\
\text { criteria (n) }\end{array}$ & 52 & 19 & 71 \\
$\begin{array}{c}\text { CT unnecessary according } \\
\text { to the medium-risk CCHR } \\
\text { criteria (n) }\end{array}$ & 260 & & \\
\hline Total (n) & 312 & 225 & 485 \\
\hline
\end{tabular}

CCHR, Canadian CT Head Rule; CT, computed tomography

When facial fractures were included as a positive head CT criterion, the sensitivity, specificity, positive predictive value (PPV), negative predictive value (NPV) and accuracy of CCHR were $92 \%, 91 \%, 90 \%, 92 \%$ and $91 \%$ 
respectively. When the positive head CT criteria included calvarial fractures and brain parenchymal and extraparenchymal findings, the sensitivity, specificity, PPV, NPV and accuracy of CCHR were 93\%, 88\%, 88\%, 94\% and $90 \%$ respectively. When bone fractures were excluded from the positive CT findings and only brain parenchymal and extra parenchymal CT findings were taken into account, the sensitivity, specificity, PPV, NPV and accuracy of CCHR were 94\%, 86\%, 84\%, 86\% and 97\% respectively.

Analysis of the diagnostic utility of CCHR according to sex revealed that the sensitivity and specificity were $94 \%$ and $87 \%$, respectively, in males (who had undergone head CT, $\mathrm{n}=233$ ) and $92 \%$ and $90 \%$, respectively, in females (who had examined with head $\mathrm{CT}, \mathrm{n}=79$ )
When the utility of CCHR was investigated in patients categorized according to their GCS scores (Table 4), the sensitivity of CCHR was better for patients with a GCS score of 14 and that specificity was higher for patients with a GCS score of 15 . The sensitivity, specificity, PPV, NPV and accuracy of CCHR were $81 \%, 91 \%, 67 \%, 96 \%$ and $90 \%$ respectively for patients with GCS score of 15 . For the patients with GCS score of 14, the sensitivity, specificity, PPV, NPV and accuracy values of CCHR were 98\%, 50\%, $93 \%, 83 \%$ and $92 \%$ respectively. The sensitivity, specificity, PPV, NPV and accuracy of CCHR were 94\%, $66 \%, 97 \%, 50 \%$ and $92 \%$ respectively for patients with GCS score of 13 .

The distribution of patients with specific head CT findings, including those with more than one CT finding, is presented in Figure 2.

Table 4. Distribution of the study patients according to the GCS scores and the CCHR.

\begin{tabular}{|c|c|c|c|c|}
\hline & $\begin{array}{l}\text { Patients with high-risk } \\
\text { CCHR criteria* }\end{array}$ & $\begin{array}{l}\text { Patients with medium- } \\
\text { risk CCHR criteria" }\end{array}$ & $\begin{array}{l}\text { Patients without any } \\
\text { CCHR risk criteria }\end{array}$ & Total (n) \\
\hline GCS 15 (n) & 22 & 47 & 362 & 431 \\
\hline GCS 14 (n) & 55 & 22 & 8 & 85 \\
\hline GCS 13 (n) & 34 & 2 & 4 & 40 \\
\hline Total (n) & 111 & 71 & 374 & 556 \\
\hline
\end{tabular}

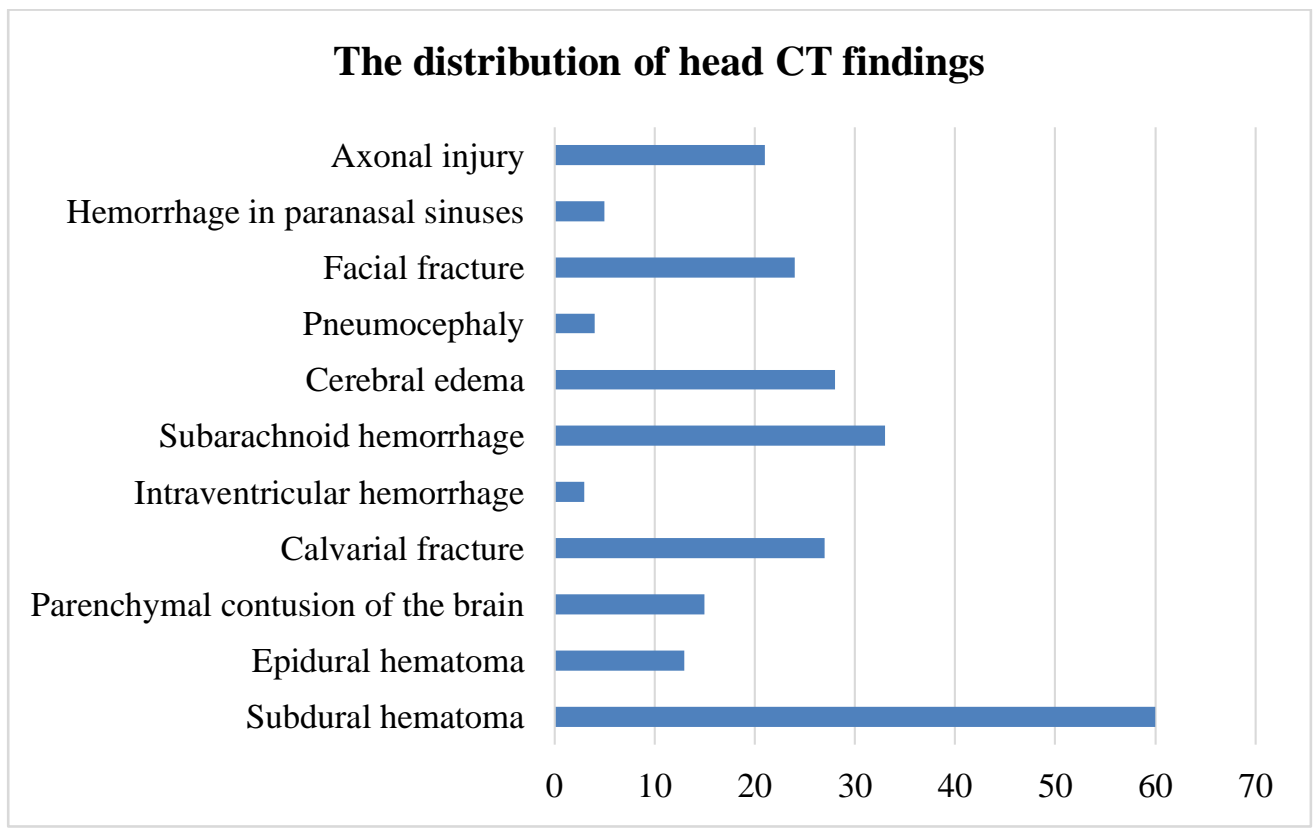

Figure 2. Frequencies of specific head CT findings within the study population Some patients had more than one positive CT finding.

Occupants in 4-wheel vehicle accidents constituted the majority of patients with minor head traumas who did not require head CT scans according to CCHR; falling accidents were the most common injury mechanism among patients who required head CTs based on CCHR (Figure 3). When the patients were categorized into three groups as those not requiring a head CT, those with at least one highrisk CCHR criterion, and those with at least one mediumrisk CCHR criterion and no high-risk CCHR criterion, there was a significant difference in the injury mechanisms among the three groups $(\mathrm{p}<0.0001$; Table 5, Figure 4).

The distribution of the injury mechanisms was also significantly different among patients with different GCS scores $(p=0.011)$. Majority of the patients with a GCS score of 15 were occupants in a 4-wheel vehicle, and falling accidents constituted the most frequent injury mechanism in patients with GCS scores of 13 and 14 (Figure 5). 


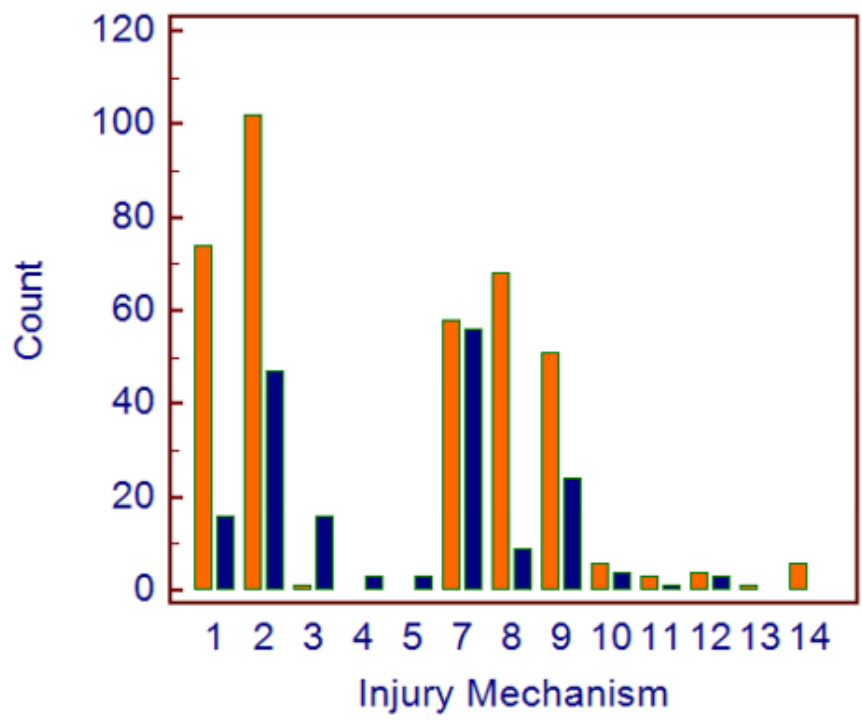

No $C T$ is required according to CCHR

CT is required according to CCHR

(1) driver in a 4 wheel motor vehicle

(2) occupant in a 4 wheel vehicle

(3) pedestrian stuck by 4 wheel motor vehicle

(4) driver in a 2 or 3 wheel motor vehicle

(5) occupant in a 2 or 3 wheel vehicle

(6) pedestrian stuck by 2 or 3 wheel motor vehicle

(7) fall accident

(8) assault by fist

(9) assault by hard object

(10) hard object fallen onto head

(11) head crash onto hard object

(12) animal attack onto head

(13) gunshots

(14) assault by sudden push or shake

Figure 3. Frequencies of specific injury mechanisms based on the need for a head CT according to the CCHR.

Table 5. Distribution of injury mechanisms according to the risk levels and requirement for a head CT based on the CCHR.

\begin{tabular}{|c|c|c|c|c|c|}
\hline Injury mechanism & $\begin{array}{c}\text { Patients with } \\
\text { high-risk } \\
\text { CCHR } \\
\text { criteria* }\end{array}$ & $\begin{array}{c}\text { Patients } \\
\text { with } \\
\text { medium- } \\
\text { risk CCHR } \\
\text { criteria }^{\#}\end{array}$ & $\begin{array}{c}\text { Patients } \\
\text { requiring } \\
\text { head CT } \\
\text { according to } \\
\text { the CCHR }\end{array}$ & $\begin{array}{c}\text { Patients } \\
\text { without any } \\
\text { CCHR risk } \\
\text { criteria }\end{array}$ & Total \\
\hline (1) Driver in a 4-wheel motor vehicle & 8 & 7 & 15 & 75 & $90(16.2 \%)$ \\
\hline (2) Occupant in a 4-wheel motor vehicle & 30 & 17 & 47 & 102 & $149(26.8 \%)$ \\
\hline (3) Pedestrian stuck by 4-wheel motor vehicle & 2 & 14 & 16 & 1 & $17(3.1 \%)$ \\
\hline $\begin{array}{l}\text { (4) Driver fallen or ejected in a } 2 \text { - or 3-wheel } \\
\text { motor vehicle }\end{array}$ & 2 & 1 & 3 & 0 & $3(0.5 \%)$ \\
\hline $\begin{array}{l}\text { (5) Occupant fallen or ejected from a 2- or 3- } \\
\text { wheel vehicle }\end{array}$ & 1 & 2 & 3 & 0 & $3(0.5 \%)$ \\
\hline $\begin{array}{l}\text { (6) Pedestrian stuck by a 2- or 3-wheel motor } \\
\text { vehicle }\end{array}$ & 0 & 0 & 0 & 0 & $0(0.0 \%)$ \\
\hline (7) Fall accident & 32 & 25 & 57 & 57 & $114(20.5 \%)$ \\
\hline (8) Assault by fist & 9 & 0 & 9 & 68 & $77(13.8 \%)$ \\
\hline (9) Assault by hard object & 21 & 3 & 24 & 51 & $75(13.5 \%)$ \\
\hline (10) Hard object fallen onto head & 2 & 2 & 4 & 6 & $10(1.8 \%)$ \\
\hline (11) Head crash onto hard object & 0 & 1 & 1 & 3 & $4(0.7 \%)$ \\
\hline (12) Animal attack onto head & 2 & 1 & 3 & 4 & $7(1.3 \%)$ \\
\hline (13) Gunshots & 0 & 0 & 0 & 1 & $1(0.2 \%)$ \\
\hline (14) Assault by sudden push or shake & 0 & 0 & 0 & 6 & $6(1.1 \%)$ \\
\hline Total & $109(19.6 \%)$ & $73(13.1 \%)$ & $182(32.7 \%)$ & $374(67.3 \%)$ & $556(100 \%)$ \\
\hline
\end{tabular}

*Patients with at least one high-risk CCHR criterion

\#Patients with at least one medium-risk CCHR criterion and had no high-risk CCHR criteria

CCHR, Canadian CT Head Rule; CT, computed tomography 


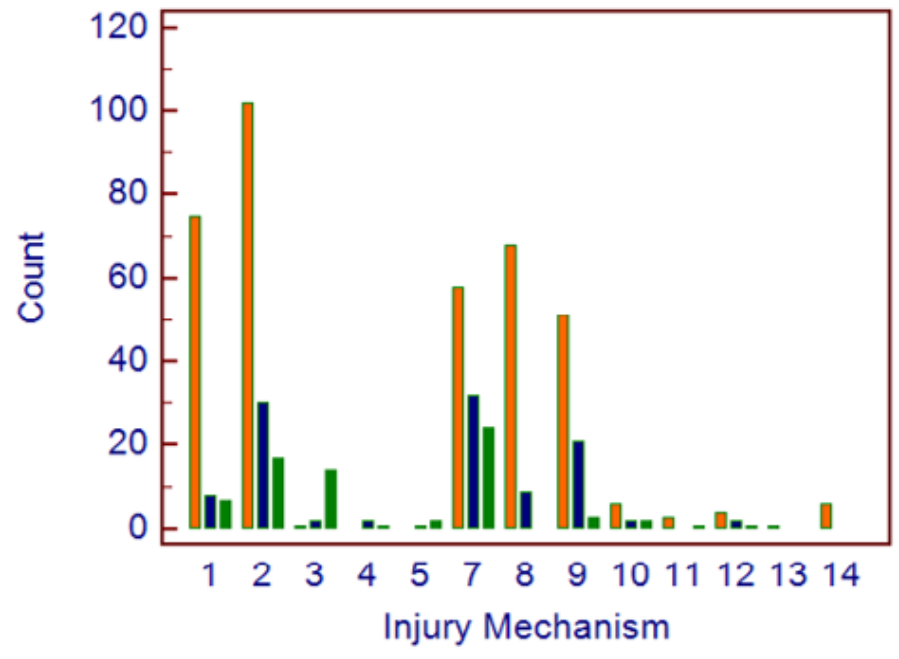

No CT is required according to CCHR

Patients with (at least 1) high risk criterion

Patients with (at least 1) medium risk criterion

(1) driver in a 4 wheel motor vehicle

(2) occupant in a 4 wheel vehicle

(3) pedestrian stuck by 4 wheel motor vehicle

(4) driver in a 2 or 3 wheel motor vehicle

(5) occupant in a 2 or 3 wheel vehicle

(6) pedestrian stuck by 2 or 3 wheel motor vehicle

(7) fall accident

(8) assault by fist

(9) assault by hard object

(10) hard object fallen onto head

(11) head crash onto hard object

(12) animal attack onto head

(13) gunshots

(14) assault by sudden push or shake

Figure 4. Frequencies of specific injury mechanism grouped based on the need of a head CT according to the high-risk and medium-risk CCHR criteria.
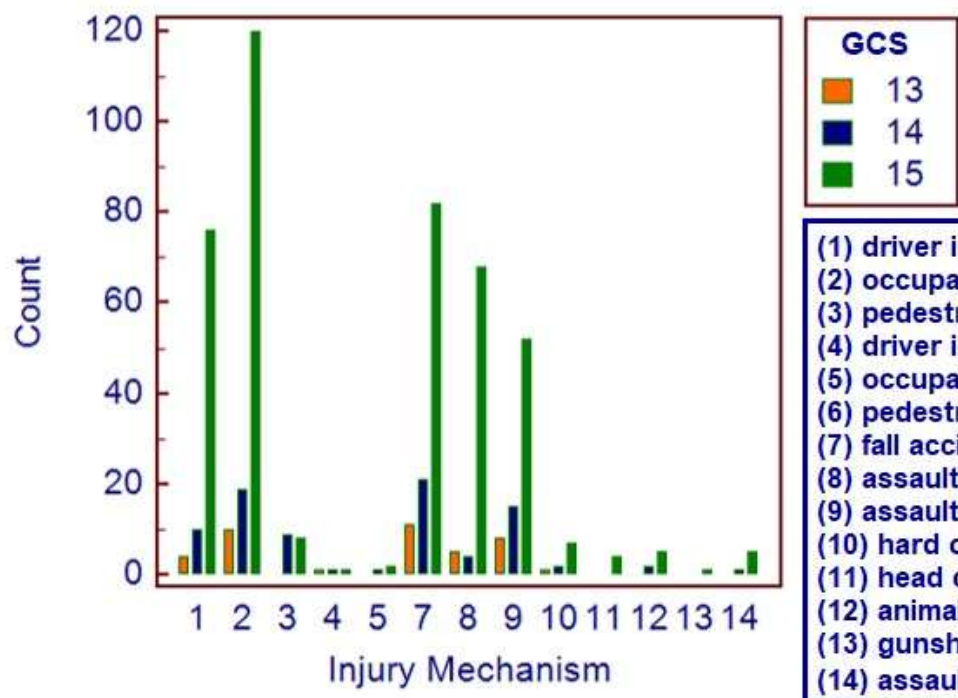

(1) driver in a 4 wheel motor vehicle

(2) occupant in a 4 wheel vehicle

(3) pedestrian stuck by 4 wheel motor vehicle

(4) driver in a 2 or 3 wheel motor vehicle

(5) occupant in a 2 or 3 wheel vehicle

(6) pedestrian stuck by 2 or 3 wheel motor vehicle

(7) fall accident

(8) assault by fist

(9) assault by hard object

(10) hard object fallen onto head

(11) head crash onto hard object

(12) animal attack onto head

(13) gunshots

(14) assault by sudden push or shake

Figure 5. Frequencies of specific injury mechanisms based on the Glasgow Coma Scale score.

The CT findings in patients who underwent head CT, although they did not require one according to CCHR, included subdural hematoma, epidural hematoma, calvarial fractures, cerebral edema, and facial fractures. In this group, subdural hematoma was the most common finding and subarachnoid hemorrhage and cerebral edema were the most common combination of CT findings in the same patient.

\section{Discussion}

The findings of the current study underline the high sensitivity and specificity of CCHR in proper detection of patients with minor head trauma for CT evaluation. The detailed analyses indicate that CCHR was highly accurate for both sexes and remained highly sensitive and specific for different injury mechanisms and GCS scores in patients with minor head traumas.

Head CT plays a very important role in many acute diseases. It has been known that in acute ischemic stroke, neuronal damage and necrosis progresses slowly. ${ }^{8} \mathrm{CT}$ might not always be effective in hyperacute stage of stroke but it is still a very useful tool to detect ischemia of brain tissue. In addition, head CT is a very important diagnostic tool that aids in the rapid diagnosis of neurotraumas and is relatively inexpensive in comparison with magnetic resonance imaging. ${ }^{1}$ Brain parenchyma as well as bone lesions and fractures can be assessed with head CT. 9 However, head CT has several disadvantages including radiation exposure and increased economic burden associated with unnecessary CT evaluations. ${ }^{1,2,4,5,10}$ Therefore, albeit the necessity of CT for major brain trauma cases, criteria for its requirement in minor head traumas remain unclear. While any serious injuries that might affect the patient's quality of life after the trauma, overuse of head CTs, and excessive radiation exposure should be avoided, clear guidelines and methods to be implemented in the emergency department are necessary.

The New Orleans criteria (NOC) by Haydel et al. is applicable only to patients with a GCS score of 15, and patients with any of the following are recommended to 
undergo a head CT scan: headache, vomiting, age above 60 years, drug or alcohol intoxication, persistent

anterograde amnesia (i.e., deficits in short-term memory), visible trauma above the clavicle, and seizure. ${ }^{11}$ This study focused on $\mathrm{CCHR},{ }^{4}$ which can be used for minor head trauma in patients with GCS scores between 13 and 15, and evaluated its diagnostic power to determine patients with minor head trauma who should be evaluated with head CT.

Mata-Mbemba et al. compared CCHR and NOC in 142 patients to identify important CT findings among patients with GCS scores from 13 to 15 and found that CCHR had lower sensitivity, higher specificity, and higher accuracy than NOC (sensitivity, $89.8 \%$ vs. $97.9 \%$; specificity, $24.7 \%$ vs. $9.7 \%$; and accuracy, $47.2 \%$ vs. $40.1 \%) .{ }^{12}$ In this study, the overall performance of CCHR was superior to that of NOC in patients with minor head trauma. A study by Kavalci et al. comparing CCHR and NOC reported that the sensitivity, specificity, PPV and NPV of CCHR were $100 \%, 0 \%, 42 \%$, and $100 \%$, respectively, in patients with a GCS score of 13. They also showed that CCHR sensitivity and specificity in patients with GCS scores of 14 and 15 were $78.5 \%$ and $42.8 \%$, respectively, and that NOC sensitivity and specificity were $85.7 \%$ and $0.7 \%$, respectively, in the same patient group. PPV and NPV were higher for CCHR than NOC (11.1\% vs. $0.7 \%$ and $95.6 \%$ vs. $84.6 \%$, respectively in the same patient population). ${ }^{13}$ In the current study, the sensitivity, specificity, PPV, NPV, and the accuracy values of CCHR (93\%, 88\%, 88\%, 94\%, and $90 \%$, respectively) were higher than those reported in the literature.

Klang et al. used CCHR to investigate the overuse of head CT scans for minor head trauma and included facial fractures as a positive CT finding, which was similar to our approach. They found that the sensitivity and NPV of the modified CCHR were both $100 \%$ for detecting brain hemorrhages, skull fractures, and face fractures. ${ }^{6}$ The current study revealed that the sensitivity, specificity, PPV, and NPV were $92 \%, 91 \%, 90 \%$, and $92 \%$ for patients with the modified CCHR, including facial fractures.

In the current study, motor vehicle accidents were more common compared with falling accidents, in agreement with majority of the literature, which reported motor vehicle accidents as the most common mechanism of head trauma. ${ }^{14,15}$ Whereas falling accidents were reported as the

most common cause of head trauma by some other studies. ${ }^{16}$ Moreover, similar to previous studies, head trauma was more frequent in males than in females in the current study. ${ }^{14-16}$ Some of the proposed underlying factors to contribute to this finding are violence, a more active city social life, working in more dangerous jobs, and higher rate of male drivers. ${ }^{13}$

The present study has several limitations that should be discussed. First, the accuracy of CCHR was assessed using the radiology reports of head CT scans as the gold standard. Although CT is highly effective in head traumas, neurological follow-ups and results of the patients were not included while assessing the sensitivity and specificity and only radiological results were included in the accuracy assessment. Additionally, CT might lead to overestimation, affecting the results of the study. Second, this was a singlecenter study, including one emergency department, and might not reflect the entire population owing to the fact that the characteristics of the local population and the patients, distribution of the injury mechanisms, study area, and internal hospital protocols may vary. Third, although injury mechanisms were grouped for careful evaluation and elucidation of the background of minor head injuries, this grouping might not reflect the exact mechanisms of trauma. For example, the group including occupants in 4-wheel motor vehicles as patients might also include passengers who experienced a head crush injury or were ejected from the vehicle. These trauma groups may not be informative enough to elucidate the magnitude of traumatic stress and the crushing mechanism, which may be related with other factors such the crushing force, the material impacting the skull, and the angle of the force applied to the head. These limitations should be considered carefully before generalizing the results of the current study.

\section{Conclusion}

In conclusion, CCHR was useful for proper selection of minor head trauma patients for evaluation by head CT scans in this retrospective study; however, the current findings indicate that CCHR might not be utilized well in determining patients who should be evaluated by head CT, an important outcome that should be considered by emergency department clinicians.

\section{Acknowledgement}

The authors thank to Harakani State Hospital, Kars, Turkey for facilitating this research.

\section{References}

1. Haydon NB. Head injury: audit of a clinical guideline to justify head CT. J Med Imaging Radiat Oncol; 2013.57(2):161-8. DOI: 10.1111/1754-9485.12007

2. Papa L, Stiell IG, Clement CM, Pawlowicz A, Wolfram A, Braga C, et al. Performance of the canadian ct head rule and the new orleans criteria for predicting any traumatic intracranial injury on computed tomography in a united states level i trauma center. Acad Emerg Med; 2012.19:2-10. DOI: 10.1111/j.1553-2712.2011.01247.x

3. Shackford SR, Wald SL, Ross SE, Cogbill TH, Hoyt DB, Morris JA, et al. The clinical utility of computed tomographic scanning and neurologic examination in the management of patients with minor head injuries. J Trauma; 1992;33:385-94. PMID: 1404507

4. Stiell IG, Wells GA, Vandemheen K, Clement C, Lesiuk H, Laupacis A, et al. The canadian ct head rule for patients with minor head injury. Lancet; 2001.357:1391-96. DOI: $10.1016 /$ S01406736(00)04561-X

5. Smits M, Dippel DW, Nederkoorn PJ, Dekker HM, Vos PE, Kool DR, et al. Minor head injury: CT-based strategies for management-a cost-effectiveness analysis. Radiology; 2010.254:532-40. DOI: 10.1148/radiol.2541081672

6. Klang E, Beytelman A, Greenberg D, Or J, Guranda L, Konen E, et al. Overuse of head ct examinations for the investigation of minor head trauma: analysis of contributing factors. J Am Coll Radiol; 2017;14:1716. DOI: $10.1016 /$ j.jacr.2016.08.032 
7. Albers CE, von Allmen M, Evangelopoulos DS, Zisakis AK, Zimmermann H, Exadaktylos AK. What is the incidence of intracranial bleeding in patients with mild traumatic brain injury? a retrospective study in 3088 canadian ct head rule patients. Biomed Res Int; 2013.2013:453978. DOI: 10.1155/2013/453978

8. Raisa N, Sujuti H, Purnomo H, Rahayu M, Dalhar M. The influence of sampling time to serum glial fibrillary acidic protein (gfap) levels in acute stroke. Malang Neurology Journal; 2019.5:30-33. DOI: http://dx.doi.org/10.21776/ub.mnj.2019.005.01.5

9. National Clinical Guideline Centre. National Institute for Health and Clinical Excellence: guidance. Head injury: triage, assessment, investigation and early management of head injury in children, young people and adults. London, UK: National Institute for Health and Care Excellence; 2014

10. Schuur JD, Carney DP, Lyn ET, Raja AS, Michael JA, Ross NG, et al. A top-five list for emergency medicine: a pilot project to improve the value of emergency care. JAMA Intern Med; 2014.174(4):50915. DOI: 10.1001/jamainternmed.2013.12688

11. Haydel MJ, Preston CA, Mills T, Luber S, Blaudeau E, DeBlieux PM. Indications for computed tomography in patients with minor head injury. N Engl J Med; 2000.343:100-105. DOI: 10.1056/NEJM200007133430204

12. Mata-Mbemba D, Mugikura S, Nakagawa A, Murata T, Kato Y, Tatewaki Y, et al. Canadian CT head rule and New Orleans Criteria in mild traumatic brain injury: comparison at a tertiary referral hospital in Japan. SpringerPlus; 2016.5:176. DOI 10.1186/s40064-016-1781-9

13. Kavalci C, Aksel G, Salt O, Yilmaz MS, Demir A, Kavalci G, et al. Comparison of the canadian ct head rule and the new orleans criteria in patients with minor head injury. World Journal of Emergency Surgery; 2014.9:31. DOI:10.1186/1749-7922-9-31

14. Stiell IG, Clement CM, Rowe BH, Schull MJ, Brison $\mathrm{R}$, Cass D, et al. Comparison of the canadian ct head rule and the new orleans criteria in patients with minor head injury. JAMA; 2005.294(12):1511-18. DOI: 10.1001/jama.294.12.1511

15. Bouida W, Marghli S, Souissi S, Ksibi H, Methammem M, Haguiga $\mathrm{H}$, et al. Prediction value of the canadian ct head rule and the new orleans criteria for positive head ct scan and acute neurosurgical procedures in minor

head trauma: a multicenter external validation study. Ann Emerg Med; 2013.61(5):521-27. DOI: 10.1016/j.annemergmed.2012.07.016

16. Ro YS, Shin SD, Holmes JF, Song KJ, Park JO, Cho JS, et al. Comparison of clinical performance of cranial computed tomography rules in patients with minor head injury: a multicenter prospective study. Acad Emerg Med; 2011.18(6):597-604. DOI: 10.1111/j.1553-2712.2011.01094.x 